\title{
Gemeinsamkeiten zwischen Parteien und medizinischen Fachgesellschaften?
}

\section{Jürg Nadig}

Präsident der Schweizerischen Gesellschaft für Medizinische Onkologie (SGMO)
Korrespondenz:

Dr. med. Jürg Nadig MAE Facharzt für Medizinische Onkologie

und Innere Medizin FMH Bannhaldenstrasse 7

CH-8180 Bülach

Tel. 0448627300

Fax 0448627301

juerg.nadig@hin.ch
Im Herbst 2008 stellte die Deutsche Börse vorübergehend den Handel ein. Die Aktienkurse fielen frei. Die Anleger hatten das Vertrauen in die Finanzindustrie verloren. In der Schweiz übernahm die Eidgenossenschaft mit einem Federstrich der Regierung, nach Konsultation der Parteien, in einer Notaktion Wertpapiere der UBS für 60 Milliarden Franken. So viel kostet das Gesundheitswesen in der Schweiz jährlich. Die UBS war zum Klumpenrisiko für die Schweiz geworden. Sie war zu gross geworden, um unterzugehen. Der gleiche Betrag, in Photovoltaik investiert, würde reichen, das Kernkraftwerk Gösgen, das ein Viertel des Atomstromes in der Schweiz produziert, zu ersetzen, neue Technologien zu fördern, die Binnennachfrage zu stimulieren, die Auslandsabhängigkeit zu verringern und einen ökologisch nachhaltigen Umbau der Wirtschaft einzuleiten. Seitdem die UBS nun Verluste schreibt, rufen die politischen Parteien nach vermehrter Finanzierung durch den Staat. Da sie deutlich weniger finanzielle Mittel von den Banken erhalten, könnten sie ihre staatspolitischen Aufgaben nicht mehr wahrnehmen.

Anlässlich der 200-Jahr-Feier der Berner Ärztegesellschaft wurde ich eingeladen, die Frage zu diskutieren, unter welchen Bedingungen Grundversorgernetzwerke nicht zu Treibern impliziter Rationierung würden. Dank der neuen Behandlungsmöglichkeiten hat sich die Überlebenszeit beim Dickdarmkrebs von sechs auf 24 Monate vervierfacht. Die Lebensverlängerung und die damit verbundene längere Gabe der teuren Medikamente lassen die Kosten aber in der dritten Potenz wachsen. Da die Lebensverlängerung von der Therapiestrategie abhängt und die kostenintensiven Therapien mehr nützen (Qualität kostet ), ist der impliziten Rationierung Tür und Tor geöffnet, insbesondere, wenn in Grundversorgernetzwerken mit Budgetverantwortung der Gewinn steigt, wenn Leistungen vorenthalten werden. Behandlungsregister können die Behandlungsqualität gesundheitsökonomisch und epidemiologisch relevanter Krankheiten erfassen und so die Verteilgerechtigkeit gewährleisten. Mit diesen Registern liesse sich das Gesundheitswesen langfristig über Qualität und Vollkosten steuern. Solche Register können als Instrument der Begleitforschung dienen und die Kostenverschiebungen zwischen Ambulanz und Spitälern, bedingt durch die DRG, erfassen. Weder die Kassen- noch die Konsumentenvertreter sahen aber Finanzierungsmöglichkeiten für eine solche Forschung. Konsumentenvertreter meinten im Gespräch, dass die aufgezeigte Lebensverlängerung für die Patienten ja wenig brächte und ich sowieso im Interesse der Pharmaindustrie spreche. Dass ich mir keine Kongressreisen von der Industrie finanzieren lasse und keine Vertreter empfange, war unwesentlich. Sie interessierten sich nur dafür, ob ich an Kongressen teilnähme, die nicht von der Industrie gesponsert seien.

In den letzten Jahren sind die Aufgaben der medizinischen Fachgesellschaften in der Schweiz gewachsen. Mit 250 Mitgliedern vertreten wir nur ein halbes Prozent der Schweizer Ärzte. Dennoch ist die Aufgabenlast für Weiter- und Fortbildung, Facharztprüfungen, Visitationen der Spitäler, Akkreditierung und Tariffragen vergleichbar mit grösseren Fachgesellschaften. Die medizinischen Onkologen stehen wegen der hohen Behandlungskosten oft im politischen Rampenlicht, von den Krankenkassen drohen WZW-Verfahren. Wir liessen uns deshalb bei der Reorganisation unserer Vereinsstruktur beraten. Die Kosten für einen professionellen Sekretär wurden vom externen Berater auf etwa 180- bis 230000 Franken veranschlagt, sodass der Mitgliederbeitrag auf 1000 Franken steigen würde. Dank dem Erlös aus verpachteten Werberechten bei Kongressen betragen die Mitgliederbeiträge heute einen Bruchteil davon. Bei Kongressen ohne Industriesponsoring wären die Registrierungsgebühren deutlich höher. Unsere Fachgesellschaft müsste die Mitgliederbeiträge massiv erhöhen. Möglicherweise bestehen in der Schweiz Ähnlichkeiten zwischen der Finanzierung der politischen Parteien und der Finanzierung der medizinischen Fachgesellschaften. Vielleicht drängen sich neue Finanzierungsmöglichkeiten für die Fortbildung auf. Über die Medikamentenkosten finanziert das Zwangskollektiv der Prämienzahler die Werbekosten der Pharmaindustrie (Novartis 2007: 11 Milliarden Dollar für PR, für Forschung 6 Milliarden Dollar). Stünde ein Teil der Werbekosten über einen Pool direkt für die Weiter- und Fortbildung und die industrieunabhängige klinische Forschung zur Verfügung, stiege das Vertrauen in die Unabhängigkeit der Ärzte, und die Fortbildungsqualität würde kaum leiden. 